\title{
Bound State Solutions of the Schrödinger Equation for Generalized Morse Potential With Position Dependent Mass
}

\author{
Altuğ $\operatorname{Arda}^{1, *}$ and Ramazan Sever $^{2,+}$ \\ ${ }^{1}$ Department of Physics Education, Hacettepe University, 06800, Ankara,Turkey \\ ${ }^{2}$ Department of Physics, Middle East Technical University, 06800, Ankara,Turkey
}

\begin{abstract}
The effective mass one-dimensional Schrödinger equation for the generalized Morse potential is solved by using Nikiforov-Uvarov method. Energy eigenvalues and corresponding eigenfunctions are computed analytically. The results are also reduced to the case of constant mass. Energy eigenvalues are computed numerically for some diatomic molecules. The results are in agreement with the ones obtained before.

Keywords: Position dependent mass, Schrödinger Equation, Generalized Morse potential, Nikiforov-Uvarov method, energy eigenvalues, eigenfunctions.
\end{abstract}

PACS numbers: 03.65.Fd, 03.65.Ge

${ }^{*}$ E-mail: arda@hacettepe.edu.tr

†E-mail: sever@metu.edu.tr 


\section{INTRODUCTION}

In recent years, the effects of the coordinate-dependence of the mass on the solutions of the relativistic and/or non-relativistic wave equations have been received a great attentions[114].This is so because it has a widely range applications on different areas, for example, in the study of the semiconductors [15], or of electronic properties of quantum wells and quantum dots [16], and the impurities in crystals [17-19]. Point canonical transformation [9], deformed algebras [20], quadratic algebra method [21], path integral method [22], Lie

algebra approach [23-25], group-theoretical approach [26], and supersymmetric formalism [27] are some of the methods used in the literature to solve the wave equations for the case of constant and/or position-dependent mass (PDM) distributions.

In the present work, we study the effects of the PDM on the solutions of the Schrödinger equation (SE) for the generalized Morse potential given by

$$
V(x)=V_{1} e^{-2 \beta\left(\frac{r-r_{0}}{r_{0}}\right)}-V_{2} e^{-\beta\left(\frac{r-r_{0}}{r_{0}}\right)},
$$

where $V_{1}$, and $V_{2}$ are two parameters, and correspond to $D$, and $2 D$ in the usual Morse potential, respectively, where $D$ is the dissociation energy of the molecule, and the parameter $\beta$ is the depth of the potential. The Morse potential is one of the important exponentially varying potential which describes the interaction in diatomic molecules [28]. The Morse potential is exactly solvable for $s$-waves. However, for any $\ell$-states only numerical solutions can be obtained with different approximation techniques [29]. We intend to apply the NikiforovUvarov (NU) method [30] to find out the energy spectra and corresponding eigenfunctions of the generalized Morse potential by using a suitable coordinate-dependence mass distribution function which is finite at infinity, and also enables us to solve the SE exactly.

The work is organized as follows. In Section II, we find out the energy eigenvalues and corresponding eigenfunctions of the SE by using the NU-method in the case of PDM. We give also the results for the case of constant mass. We give the numerical results for the bound state energies of $\mathrm{H}_{2}$, and $\mathrm{LiH}$ molecules in the case of constant and spatially dependent mass in Table I. We summarize our conclusions in Section III. 


\section{NIKIFOROV-UVAROV METHOD AND BOUND STATES}

The one-dimensional time-independent effective mass SE equation with the potential $V(x)$ reads $[6]$

$$
-\frac{\hbar^{2}}{2 m(x)} \frac{d^{2} \psi(x)}{d x^{2}}+\frac{\hbar^{2}}{2}\left(\frac{m^{\prime}(x)}{m^{2}(x)}\right) \frac{d \psi(x)}{d x}+\left[V(x)+U_{\alpha \beta \gamma}(x)-E\right] \psi(x)=0,
$$

where prime denotes the derivative of mass $m(x)$ with respect to position and

$$
U_{\alpha \beta \gamma}(x)=-\frac{\hbar^{2}}{4 m^{3}(x)(a+1)}\left[(\alpha+\gamma-a) m(x) m^{\prime \prime}(x)+2(a-\alpha \gamma-\alpha-\gamma) m^{\prime 2}(x)\right]
$$

where the ambiguity parameters proposed by different authors in the literature satisfy $\alpha+$ $\beta+\gamma=-1$. In the ordering proposed by Weyl, the parameters have the values $a=1$, $\alpha=\gamma=0$. In the parameter set due to Li and Kuhn $a=\alpha=0$, and $\gamma=-\frac{1}{2}$, etc. [6]. It is found in Ref. [6] that the Weyl and Li and Kuhn ambiguity orderings are equivalent in the case of SE equation.

By redefinition of the wave function

$$
\psi(x)=\sqrt{m(x)} \phi(x)
$$

we get the SE as

$$
-\frac{\hbar^{2}}{2 m(x)} \frac{d^{2} \phi(x)}{d x^{2}}+\left(U_{e f f}(x)-E\right) \phi(x)=0 .
$$

The effective potential in the above equation is

$$
U_{e f f}(x)=U_{\alpha \beta \gamma}(x)+V(x)+\frac{\hbar^{2}}{4 m^{2}(x)}\left(\frac{3 m^{\prime 2}(x)}{2 m(x)}-m^{\prime \prime}\right) .
$$

We consider the generalized Morse potential, which can be used to describe the vibrations of a two-atomic molecule, as the following

$$
V(x)=V_{1} e^{-2 \beta x}-V_{2} e^{-\beta x},(0 \leq x \leq \infty)
$$


where $V_{1}$, and $V_{2}$ are two real parameters, and $x=\left(r-r_{0}\right)$. The parameter $\beta$ is $\alpha^{\prime} / r_{0}$, here, $\alpha^{\prime}$ is a positive parameter, and $r_{0}$ is the equilibrium distance [29].

Here, we prefer to use the following mass-distribution

$$
m(x)=\frac{m_{0}}{\left(1-\eta e^{-\beta x}\right)^{2}}
$$

where $m_{0}$ corresponds to the constant mass, and the free parameter $\eta$ satisfies the condition that $\eta<1$ to handle a positive mass at the zero. We get a finite mass distribution with this constraint on $\eta$. We can check out the results of the case of constant mass. Further, we solve the SE analytically by using such a mass-function.

Substituting Eqs. (8) and (7) into Eq. (5) we get

$$
\begin{aligned}
\frac{d^{2} \phi(x)}{d x^{2}} & +\frac{1}{\left(1-\eta e^{-\beta x}\right)^{2}}\left\{\left[2 A_{2} \beta^{2} \eta+\frac{2 m_{0} V_{2}}{\hbar^{2}}\right] e^{-\beta x}\right. \\
& \left.+\left[4 \beta^{2} \eta^{2}\left(A_{1}+A_{2}\right)-\frac{2 m_{0} V_{1}}{\hbar^{2}}\right] e^{-2 \beta x}+\frac{2 m_{0} E}{\hbar^{2}}\right\} \phi(x)=0
\end{aligned}
$$

where

$$
\begin{aligned}
& A_{1}=\frac{a-\alpha \gamma-\alpha-\gamma}{1+a}-\frac{3}{4} \\
& A_{2}=\frac{\alpha+\gamma-a}{2(1+a)}+\frac{1}{2}
\end{aligned}
$$

By using the transformation $z=e^{-\beta x}(0 \leq z \leq 1)$, we obtain

$$
\frac{d^{2} \phi(z)}{d z^{2}}+\frac{1-\eta z}{z(1-\eta z)} \frac{d \phi(z)}{d z}+\frac{1}{[\eta(1-\eta z)]^{2}}\left(-\varepsilon_{1} z^{2}-\varepsilon_{2} z-\epsilon_{n \ell}\right) \phi(z)=0,
$$

where

$$
\begin{aligned}
-\varepsilon_{1} & =4 \eta^{2}\left(\frac{a-2 \alpha \gamma-\alpha-\gamma}{2(1+a)}-\frac{1}{4}\right)-\frac{2 m_{0} V_{1}}{\beta^{2} \hbar^{2}} \\
-\varepsilon_{2} & =\eta\left(\frac{\alpha+\gamma+1}{1+a}\right)+\frac{2 m_{0} V_{2}}{\beta^{2} \hbar^{2}} \\
-\epsilon_{n \ell} & =\frac{2 m_{0} E}{\beta^{2} \hbar^{2}}
\end{aligned}
$$


Now to apply the NU-method [30], we rewrite Eq. (11) in the following form

$$
\phi^{\prime \prime}(z)+\frac{\tilde{\tau}(z)}{\sigma(z)} \phi^{\prime}(z)+\frac{\tilde{\sigma}(z)}{\sigma^{2}(z)} \phi(z)=0
$$

where $\sigma(z)$ and $\tilde{\sigma}(z)$ are polynomials with second-degree, at most, and $\tilde{\tau}(z)$ is a polynomial with first-degree. We define the total wave function as

$$
\phi(z)=\xi(z) \psi(z)
$$

Thus Eq. (13) is reduced to a hypergeometric type equation

$$
\sigma(z) \psi^{\prime \prime}(z)+\tau(z) \psi^{\prime}(z)+\lambda \psi(z)=0
$$

We also define the new eigenvalue for the Eq. (13) as

$$
\lambda=\lambda_{n}=-n \tau^{\prime}-\frac{n(n-1)}{2} \sigma^{\prime \prime},(n=0,1,2, \ldots)
$$

where

$$
\tau(z)=\tilde{\tau}(z)+2 \pi(z)
$$

The derivative of $\tau(z)$ must be negative. $\lambda\left(\lambda_{n}\right)$ is obtained from a particular solution of the polynomial $\psi_{n}(z)$ with the degree of $n . \psi_{n}(z)$ is the hypergeometric type function whose solutions are given by [30]

$$
\psi_{n}(z)=\frac{b_{n}}{\rho(z)} \frac{d^{n}}{d y^{n}}\left[\sigma^{n}(z) \rho(z)\right]
$$

where the weight function $\rho(z)$ satisfies the equation

$$
\frac{d}{d z}[\sigma(z) \rho(z)]=\tau(z) \rho(z)
$$

On the other hand, the function $\xi(z)$ satisfies the relation 


$$
\xi^{\prime}(z) / \xi(z)=\pi(z) / \sigma(z)
$$

Comparing Eq. (11) with Eq. (13), we have

$$
\tilde{\tau}(z)=1-\eta z, \quad \sigma(z)=z(1-\eta z), \quad \tilde{\sigma}(z)=-\varepsilon_{1} z^{2}-\varepsilon_{2} z-\epsilon_{n \ell}
$$

The $\pi(z)$ has the form [30]

$$
\pi(z)=\frac{\sigma^{\prime}(z)-\tilde{\tau}(z)}{2} \pm \sqrt{\left(\frac{\sigma^{\prime}(z)-\tilde{\tau}(z)}{2}\right)^{2}-\tilde{\sigma}(z)+k \sigma(z)}
$$

or, explicitly

$$
\pi(z)=-\frac{\eta z}{2} \pm \sqrt{\left(\frac{\eta^{2}}{4}-k \eta+\varepsilon_{1}\right) z^{2}+\left(\varepsilon_{2}+k\right) z+\epsilon_{n \ell}} .
$$

The constant $k$ is determined by imposing a condition such that the discriminant under the square root should be zero. The roots of $k$ are $k_{1,2}=-\varepsilon_{2}-2 \eta \epsilon_{n \ell} \mp \sqrt{\epsilon_{n \ell}} A$, where $A=\sqrt{4 \eta^{2} \epsilon_{n \ell}+4 \eta \varepsilon_{2}+\eta^{2}+4 \varepsilon_{1}}$.

Substituting these values into Eq. (23), we get for $k_{1}$

$$
\pi(z)=-\frac{\eta z}{2} \mp\left[\left(\frac{A}{2}-\eta \sqrt{\epsilon_{n \ell}}\right) z+\sqrt{\epsilon_{n \ell}}\right]
$$

and for $k_{2}$

$$
\pi(z)=-\frac{\eta z}{2} \mp\left[\left(\frac{A}{2}+\eta \sqrt{\epsilon_{n \ell}}\right) z-\sqrt{\epsilon_{n \ell}}\right]
$$

Now we calculate the polynomial $\tau(z)$ from $\pi(z)$ such that its derivative with respect to $z$ must be negative. Thus we take the root $k_{2}$, and by using Eq. (25) for $\pi(z)$ we get

$$
\tau(z)=1+2 \sqrt{\epsilon_{n \ell}}-2\left(\frac{A}{2}+\eta \sqrt{\epsilon_{n \ell}}+\eta\right) z
$$

with the derivative $\tau^{\prime}(z)=-2\left(\frac{A}{2}+\eta \sqrt{\epsilon_{n \ell}}+\eta\right)$. 
The constant $\lambda=k+\pi^{\prime}(z)$ becomes

$$
\lambda=-\varepsilon_{2}-2 \eta \epsilon_{n \ell}-\sqrt{\epsilon_{n \ell}}(A+\eta)-\frac{1}{2}(A+\eta),
$$

and Eq. (16) gives us

$$
\lambda_{n}=2 n\left(\frac{A}{2}+\eta \sqrt{\epsilon_{n \ell}}+\eta\right)+\eta n(n-1) .
$$

Substituting the values of the parameters given by Eq. (12), and setting $\lambda=\lambda_{n}$, one can find the energy eigenvalues as

$$
\epsilon_{n \ell}=\left\{\frac{\left[n^{2}+n-(1 / 2)\right] \eta-\varepsilon_{2}-2[n+(1 / 2)] \sqrt{\varepsilon_{1}-\left(\eta^{2} / 2\right)}}{(2 n+1) \eta-\sqrt{4 \varepsilon_{1}-2 \eta^{2}}}\right\}^{2} .
$$

It is seen that there is a strong dependence of the energy eigenvalues to the parameter $\eta$. Further, one can see that the generalized Morse potential has a real energy spectra in the case of PDM under the condition that

$$
\frac{m_{0} V_{1}}{\beta^{2} \hbar^{2}}>\eta^{2}\left(\frac{a-2 \alpha \gamma-\alpha-\gamma}{1+a}-\frac{1}{4}\right)
$$

We get the following energy spectra in constant mass case as

$$
\epsilon_{n \ell}^{\eta=0}=\frac{1}{4}\left[2 n+1+\frac{\varepsilon_{2}}{\sqrt{\varepsilon_{1}}}\right]^{2} .
$$

It is exactly same result obtained in the literature [29].

We list the numerical results for the bound state energies of the $\mathrm{H}_{2}$, and $\mathrm{LiH}$ molecules in the constant mass case in Table I. We use the same parameters given in Ref. (29), such as $D, r_{0}, m_{0}, \alpha^{\prime}$, and $E_{0}$, to compare our results. Here, the new parameter $E_{0}$ is a short notation, i.e., $\hbar^{2} /\left(m_{0} r_{0}^{2}\right)$. We also give the bound state energies of the above molecules for three different values of $\eta$ for each molecule in Table I. For simplicity, we choose the Weyl ordering for the ambiguity parameters in the numerical analyze.

Now let us find the eigenfunctions. We first compute the weight function from Eqs. (17) and (19) 


$$
\rho(z)=z^{-2 \sqrt{\epsilon_{n \ell}}}(1-\eta z)^{\tilde{A}}
$$

where $\tilde{A}=\sqrt{1+4 \epsilon_{n \ell}+\frac{4}{\eta}\left(\varepsilon_{2}+\frac{\varepsilon_{1}}{\eta}\right)}$, and the wave functions become

$$
\psi_{n}(z)=\frac{b_{n}}{z^{-2 \sqrt{\epsilon_{n \ell}}}(1-\eta z)^{\tilde{A}}} \frac{d^{n}}{d z^{n}}\left[z^{n-2 \sqrt{\epsilon_{n \ell}}}(1-\eta z)^{n+\tilde{A}}\right] .
$$

where $b_{n}$ is a normalization constant. In the limit $\eta \rightarrow 1$, the polynomial solutions can be written in terms of the Jacobi polynomials with weight function $\rho(z)$ as [31]

$$
\psi_{n}(z) \simeq P_{n}^{\left(\tilde{A},-2 \sqrt{\epsilon_{n \ell}}\right)}(2 \eta z-1), \quad \tilde{A}>-1,-2 \sqrt{\epsilon_{n \ell}}>-1
$$

On the other hand, the other part of the wave function is obtained from the Eq. (20) as

$$
\xi(z)=z^{-\sqrt{\epsilon_{n \ell}}}(1-\eta z)^{(1 / 2)(1+\tilde{A})} .
$$

Thus, the total eigenfunctions take

$$
\phi_{n}(z)=b_{n}^{\prime} z^{-\sqrt{\epsilon_{n \ell}}}(1-\eta z)^{(1 / 2)(1+\tilde{A})} P_{n}^{\left(\tilde{A},-2 \sqrt{\epsilon_{n \ell}}\right)}(2 \eta z-1) .
$$

where $b_{n}^{\prime}$ is the new normalization constant. The normalization condition $\int_{0}^{1}|\phi(z)|^{2} d z=1$ gives us

$$
b_{n}^{\prime 2}\left(\frac{1}{2}\right)^{\tilde{A}-2 \sqrt{\epsilon_{n \ell}}} \int_{-1}^{+1}(1-x)^{1+\tilde{A}}(1+x)^{-2 \sqrt{\epsilon_{n \ell}}} P_{n}^{\left(\tilde{A},-2 \sqrt{\epsilon_{n \ell}}\right)}(x) P_{m}^{\left(\tilde{A},-2 \sqrt{\epsilon_{n \ell}}\right)}(x) d x=1,
$$

where we use a new variable defined as $x=2 \eta z-1$. By using the required identities of the Jacobi polynomials [32, 33], we obtain

$$
\begin{aligned}
b_{n}^{\prime 2} & =\frac{2^{2 \tilde{A}-4 \sqrt{\epsilon_{n \ell}}+1}}{\tilde{A}-2 \sqrt{\epsilon_{n \ell}}+2 n+1} \frac{2\left(\tilde{A}-\sqrt{\epsilon_{n \ell}}\right)\left(1-\sqrt{\epsilon_{n \ell}}+2 n\right)+4 n(1+n)}{\left(\tilde{A}-2 \sqrt{\epsilon_{n \ell}}+2 n+2\right)\left(\tilde{A}-2 \sqrt{\epsilon_{n \ell}}+2 n\right)} \\
& \times \frac{\Gamma(\tilde{A}+n+1) \Gamma\left(-2 \sqrt{\epsilon_{n \ell}}+n+1\right)}{n ! \Gamma\left(\tilde{A}-2 \sqrt{\epsilon_{n \ell}}+n+1\right)} .
\end{aligned}
$$




\section{CONCLUSION}

We have solved the one-dimensional position-dependent effective mass SE for the generalized Morse potential by using NU-method and obtained analytically the energy eigenvalues and corresponding eigenfunctions. They depend on the free parameter $\eta$ strongly. We have shown that the results can be reduced to the ones obtained for the case of the constant mass. We have listed the numerical values of the energy eigenvalues in Table I for the $H_{2}$, and $\mathrm{LiH}$ molecules for different values of the quantum number $n$, and free parameter $\eta$.

\section{ACKNOWLEDGMENTS}

This research was partially supported by the Scientific and Technical Research Council of Turkey. 
[1] O. von Roos, Phys. Rev. B 27, 7547 (1983).

[2] D. J. BenDaniel, and C. B. Duke, Phys. Rev. 152, 683 (1966).

[3] J. M. LevyLeblond, Phys. Rev. A 52, 1845 (1995).

[4] L. Dekar, L. Chetouani, and T. F. Hammann, J. Math. Phys. 39, 2551 (1998).

[5] L. Dekar, L. Chetouani, and T. F. Hammann, Phys. Rev. A 59, 107 (1999).

[6] A. S. Dutra, and C. A. S. Almeida, Phys. Lett. A 275, 25 (2000).

[7] B. Gonul, B. Gonul, D. Tutcu, and O. Ozer, Mod. Phys. Lett. A 17, 2057 (2002).

[8] B. Gonul, O. Ozer, B. Gonul, and F. Uzgun, Mod. Phys. Lett. A 17, 2453 (2002).

[9] A. D. Alhaidari, Phys. Rev. A 66, 042116 (2002).

[10] B. Roy, and P. Roy, J. Phys. A: Math. Gen. 35, 3691 (2002).

[11] C. Quesne, and V. M. Tkachuk, J. Phys. A: Math. Gen. 36, 10373 (2003).

[12] C. Quesne, and V. M. Tkachuk, J. Phys. A: Math. Gen. 37, 10095 (2004).

[13] B. Bagchi, A. Banerjee, C. Quesne, and V. M. Tkachuk, J. Phys. A: Math. Gen. 38, 2929 (2005).

[14] C. Tezcan, and R. Sever, J. Math. Chem. 42, 387 (2007) [arXiv: quant-ph/0604041].

[15] T. Gora, and F. Williams, Phys. Rev. 177, 11979 (1969).

[16] L. Serra, and E. Lipparini, Europhys. Lett. 40, 667 (1997).

[17] G. H. Wanner, Phys. Rev. 52, 191 (1957).

[18] J. M. Luttinger and W. Kohn, Phys. Rev. 97, 869 (1955).

[19] J. C. Slater, Phys. Rev. 76, 1592 (1949).

[20] C. Quesne, and V. M. Tkachuk, J. Phys. A: Math. Gen. 37, 10095 (2004) [arXiv: math-ph/0403047].

[21] C. Quesne, SIGMA3, 067 (2007) [arXiv: math-ph/0705.2577].

[22] H. Kleinert, Path Integrals in quantum mechanics statistics and polymer physics, 2nd ed. , (WorldScientific, 1995).

[23] J. Wu, Y. Alhassid, and F. Gursey, Ann. Phys. 196, 163 (1989).

[24] J. Wu, and Y. Alhassid, J. Math. Phys. 31, 557 (1990).

[25] G. Levai, J. Phys. A: Math. Gen. 27, 3809 (1994).

[26] S. A. Yahiaoui, and M. Bentaiba, arXiv:0803.4376 math-ph]. 
[27] B. Bagchi, and T. Tanaka, Phys. Lett. A 372, 5390 (2008).

[28] S. Flügge, Practical Quantum Mechanics I , (Springer-Verlag, 1971).

[29] I. Nasser, M. S. Abdelmonem, H. Bahlouli, and A. D. Alhaidari, J. Phys. B 40.21, 4245 (2007), and references therein.

[30] A. F. Nikiforov, and V. B. Uvarov, Special Functions of Mathematical Physics , (Birkhäuser, Basel, 1988).

[31] C. W. Wong, Introduction to Mathematical Physics-Methods and Concepts, (Oxford University Press, 1991).

[32] W. W. Bell, Special Functions for Scientists, and Engineers (Dover Publications, Mineola, N. Y., 1968).

[33] I. S. Gradshteyn, and I. M. Ryzhik, Table of Integrals, Series, and Poducts, edited by A. Jeffrey (Academic Press, 1980). 
TABLE I: The dependence of the bound states on $n$ in $\mathrm{eV}$ for $H_{2}\left(D=4,7446 \mathrm{eV}, r_{0}=0,7416 \AA\right.$, $m_{0}=0,50391 \mathrm{amu}, \alpha^{\prime}=1,440558$, and $\left.E_{0}=1,508343932 \times 10^{-2} \mathrm{eV}\right)$, and $\mathrm{LiH}$ molecules $(D=$ $2,515287 \mathrm{eV}, r_{0}=1,5956 \AA$ A $m_{0}=0,8801221 \mathrm{amu}, \alpha^{\prime}=1,7998368$, and $E_{0}=1,865528199 \times$ $\left.10^{-3} \mathrm{eV}\right)[29]$.

\begin{tabular}{|c|c|c|c|c|c|c|}
\hline & & & & & & $\eta=0$ \\
\hline$n$ & $E_{n}\left(H_{2}\right)^{a}$ & $E_{n}\left(H_{2}\right)^{b}$ & & & $E_{n}(L i H)^{a}$ & $E_{n}(L i H)^{b}$ \\
\hline 0 & -4.476 & -4.476 & & & -2.429 & -2.429 \\
\hline 2 & -3.480 & -3.480 & & & -2.098 & -2.098 \\
\hline 4 & -2.609 & -2.609 & & & -1.792 & -1.792 \\
\hline 10 & -0.748 & -0.748 & & & -1.018 & -1.018 \\
\hline 15 & -0.057 & & & & -0.539 & \\
\hline \multirow[t]{2}{*}{20} & & & & & -0.211 & -0.211 \\
\hline & & & & & & $\eta \neq 0$ \\
\hline$n$ & $E_{n}\left(H_{2}\right)^{c}$ & $E_{n}\left(H_{2}\right)^{d}$ & $E_{n}\left(H_{2}\right)^{e}$ & $E_{n}(L i H)^{c}$ & $E_{n}(L i H)^{d}$ & $E_{n}(L i H)^{e}$ \\
\hline 0 & -4.528 & -4.582 & -4.637 & -2.446 & -2.463 & -2.481 \\
\hline 2 & -3.706 & -3.955 & -4.228 & -2.176 & -2.259 & -2.346 \\
\hline 4 & -2.953 & -3.363 & -3.856 & -1.920 & -2.062 & -2.219 \\
\hline 6 & -2.274 & -2.809 & -3.522 & -1.677 & -1.872 & -2.099 \\
\hline 10 & -1.152 & -1.818 & -2.985 & -1.233 & -1.512 & -1.880 \\
\hline 15 & -0.251 & -0.824 & -2.644 & -0.763 & -1.105 & -1.653 \\
\hline 20 & -0.012 & -0.169 & & -0.395 & -0.748 & -1.486 \\
\hline
\end{tabular}

${ }^{a}$ our results

${ }^{b}$ results obtained in Ref [29]

${ }^{c}$ results for $\eta=0.2$

${ }^{d}$ results for $\eta=0.4$

${ }^{e}$ results for $\eta=0.6$ 